\title{
Corrigendum: Fast native-SAD phasing for routine macromolecular structure determination
}

Tobias Weinert, Vincent Olieric, Sandro Waltersperger, Ezequiel Panepucci, Lirong Chen, Hua Zhang, Dayong Zhou, John Rose, Akio Ebihara, Seiki Kuramitsu, Dianfan Li, Nicole Howe, Gisela Schnapp, Alexander Pautsch, Katja Bargsten, Andrea E Prota, Parag Surana, Jithesh Kottur, Deepak T Nair, Federica Basilico, Valentina Cecatiello, Sebastiano Pasqualato, Andreas Boland, Oliver Weichenrieder, Bi-Cheng Wang, Michel O Steinmetz, Martin Caffrey \& Meitian Wang

Nat. Methods 12, 131-133 (2015); published online 15 December 2014; corrected after print 6 February 2015

In the version of this article initially published, the Hendrickson formula in the Figure 2 legend incorrectly had $\left(2 N_{\mathrm{A}} / N_{\mathrm{P}}\right)^{1 / 2}$ divided by $\left(f^{\prime \prime} / Z_{\text {eff }}\right)$; these terms should have been multiplied. The error has been corrected in the HTML and PDF versions of the article.

\section{Corrigendum: Simultaneous all-optical manipulation and recording of neural circuit activity with cellular resolution in vivo}

\author{
Adam M Packer, Lloyd E Russell, Henry W P Dalgleish \& Michael Häusser \\ Nat. Methods 12, 140-146 (2015); published online 22 December 2014; corrected after print 6 February 2015
}

In the version of this article initially published, the size of the scale bar reported in the legend of Figure 3a was incorrect. The correct size is $100 \mu \mathrm{m}$, not $50 \mu \mathrm{m}$. In addition, the volume of injected virus in the Online Methods section "Titration of calcium indicator expression" had the incorrect unit. The correct volume is $100 \mathrm{nl}$, not $100 \mu \mathrm{l}$. The errors have been corrected in the HTML and PDF versions of the article.

\section{Corrigendum: Fixation-resistant photoactivatable fluorescent proteins for CLEM}

Maria G Paez-Segala, Mei G Sun, Gleb Shtengel, Sarada Viswanathan, Michelle A Baird, John J Macklin, Ronak Patel, John R Allen, Elizabeth S Howe, Grzegorz Piszczek, Harald F Hess, Michael W Davidson, Yalin Wang \& Loren L Looger

Nat. Methods 12, 215-218 (2015); published online 12 January 2015; corrected after print 4 May 2015

In the version of this article initially published, scale bars that had been erroneously inserted into Figure 1c during image processing were covered with black boxes. The error has been corrected, through removal of both the boxes and the scale bars beneath them, for the HTML and PDF versions of the article.

\section{Corrigendum: Accurate macromolecular structures using minimal measurements from $\mathrm{X}$-ray free-electron lasers}

Johan Hattne, Nathaniel Echols, Rosalie Tran, Jan Kern, Richard J Gildea, Aaron S Brewster, Roberto Alonso-Mori, Carina Glöckner, Julia Hellmich, Hartawan Laksmono, Raymond G Sierra, Benedikt Lassalle-Kaiser, Alyssa Lampe, Guangye Han, Sheraz Gul, Dörte DiFiore, Despina Milathianaki, Alan R Fry, Alan Miahnahri, William E White, Donald W Schafer, M Marvin Seibert, Jason E Koglin, Dimosthenis Sokaras, Tsu-Chien Weng, Jonas Sellberg, Matthew J Latimer, Pieter Glatzel, Petrus H Zwart, Ralf W Grosse-Kunstleve, Michael J Bogan, Marc Messerschmidt, Garth J Williams, Sébastien Boutet, Johannes Messinger, Athina Zouni, Junko Yano, Uwe Bergmann, Vittal K Yachandra, Paul D Adams \& Nicholas K Sauter Nat. Methods 11, 545-548 (2014); published online 16 March 2014; corrected after print 3 June 2015

In the version of this article initially published, the authors claimed that with the tool cctbx.xfel, weak diffraction signals can be measured using fewer crystal specimens than are needed for the previously available program CrystFEL. However, there is not enough evidence to support this claim. The inaccurate statements have been corrected in the HTML and PDF versions of the article. 